\title{
La atención a personas dependientes en Europa: conceptos actuales y perspectivas futuras
}

\author{
The attention to dependent people in Europe: current \\ concepts and future perspectives
}

\begin{abstract}
RESUMEN
Ante la constatación de importantes cambios demográficos, económicos y sociales en Europa que afectan a la provisión de servicios de apoyo a las personas dependientes, la Comisión Europea financió la investigación "La atención a personas dependientes en Europa (conceptos actuales y perspectivas futuras)" que a continuación se presenta. Entre los seis países que conforman la muestra se encuentran importantes diferencias conceptuales y estructurales en la atención a la dependencia, así como una diversidad en la tipología del personal, aunque se identifican algunos requisitos del trabajo comunes a todos los sectores de población con los que se interviene. Las conclusiones manifiestan que, salvo excepciones, el sueldo y otras condiciones laborales son insuficientes, aunque el trabajo a tiempo parcial, muy frecuente en el sector, ha permitido a la mujer conciliar mejor la vida laboral y familiar, aún con el riesgo de seguir manteniendo la excesiva feminización del sector profesional. Los trabajadores perciben que la atención que prestan tiene bajo estatus, aunque no albergan dudas sobre su importancia. Esta situación puede producir una grave escasez en la dotación de personal en los países de la U.E. Se valora la utilidad de tres medidas que pueden complementarse: la estimulación de la atención informal (permisos parentales e incentivos familiares); aprovechar bolsas de cuidadores (actuales o potenciales) infrautilizados, generalmente con bajo nivel de cualificación; revalorizar la atención a dependientes con nuevos perfiles profesionales y mejora de la cualificación, formación continua y desarrollo profesional, lo que redundaria en un mayor reconocimiento social. Todo ello sin olvidar la importancia de aumentar la diversidad en el personal desde los puntos de vista étnico y de género.
\end{abstract}




\title{
PALABRAS CLAVE
}

Dependencia en la U.E., Cuidados a personas dependientes, Perfiles profesionales de los cuidadores.

\begin{abstract}
Confronted to the verification of the important demographic, economic and social changes in Europe affecting the provision of support services to dependent persons, the European Commission has financed the research "The attention to dependent people in Europe (current concepts and future perspectives)" that is presented in this article. Among the six countries conforming the sample, we can observe conceptual and structural important differences in the attention to the dependence, as well as diversity in the personnel typology, although some work requirements are identify to be common in all the population's sectors of intervention.

The conclusions manifest that, except some exceptions, the salary and other work conditions are insufficient, although the work on partial time, very frequent in this sector, has allowed the woman to reconcile the work and family life better, still with the risk of continuing maintaining the excessive feminisation of the professional sector.

The workers perceive that the attention provided by them is considered as low status, although they don't have doubts about its importance. This situation can produce a serious shortage in the endowment of professionals in the countries of the E.U. The utility of three measures is valued as complementary: the stimulation of the informal care (family permits and incentives); to take advantage of underestimate pockets of caregivers (current or potentials), generally with low qualification levels; to revalue the attention to dependent persons with new professionals profiles and improvement of the qualification, continuous formation and professional development, redounding in a wider social recognition. Without forgetting the importance to increase the diversity of the professionals from an ethnic and gender points of view.
\end{abstract}

\section{KEY WORDS}

Dependence in the E.U, Dependent persons care, The professional profiles of caregivers. 
La atención a personas dependientes en Europa (conceptos actuales y perspectivas futuras) es el titulo de una importante investigación (Care Work) financiada por la Comisión Europea. Dicha financiación es prueba del interés de la Unión Europea en la "atención" y "prestación de ayuda" a lo largo del ciclo vital, de la infancia a la vejez, interés al que mueven objetivos de empleo. Los "servicios de ayuda" se consideran una de las condiciones necesarias para la creación de empleo femenino y el fomento de la igualdad de género, mientras que la "prestación de ayuda" como tal se vislumbra como un área importante de crecimiento futuro del empleo. Además, ambas razones hacen sitio a la atención en las politicas nacionales, en las que sin embargo caben otros asuntos como los servicios de "cuidado y atención infantil" durante los primeros años dirigidos a combatir la pobreza y fomentar el desarrollo y el rendimiento escolar de los niños de zonas deprimidas. Por falta de competencias en la infancia, la UE no contempla esta dimensión en sus políticas.

Esta perspectiva laboral (de empleo) de la UE es la razón del principal objetivo que se plantea este estudio sobre la atención a personas dependientes en Europa: contribuir al desarrollo de un empleo de calidad en los servicios que responden a las necesidades de las sociedades en rápido cambio. Volveremos más adelante sobre el sentido e implicaciones cuando hablamos de empleo "de calidad”, señalando el interés manifiesto de la UE tanto por la calidad como por el volumen del empleo. Bien es cierto que el concepto de "sociedades en rápido cambio" necesita una mayor elaboración, puesto que llama la atención sobre el contexto cambiante de la atención a dependientes, que afecta no sólo a las dimensiones y estructura del personal sino también al trabajo como tal. ¿Cuáles son estos cambios?

\section{Cambios en la oferta y la demanda}

- Mayor demanda de servicios formales por mor de los cambios demográficos, económicos y sociales (como el envejecimiento de la población o el aumento del empleo de ambos cónyuges).

- Cambios en la oferta de cuidadores, formales e informales, debido al descenso de la población de mujeres de bajo nivel educativo, el aumento del empleo femenino y los cambios demográficos que generan "familias escuálidas" (es decir, menos miembros en cada generación al disminuir el tamaño de la familia).

Cambios en la posición social de los usuarios de servicios

- El cambio en la percepción social de los usuarios de servicios, con más énfasis en su consideración como sujetos activos, ciudadanos con derechos y consumidores y en la capacitación, integración, autonomía, elección y desarrollo.

\section{Cambios en la politica de bienestar}

- Mayor énfasis en la descentralización y en los enfoques integradores y globales que entrañan colaboración y trabajo en red, combinación de prestaciones, elección y flexibilidad.

- Límites cada vez más imprecisos entre los campos hasta ahora distintos de las políticas y de la prestación.

- Mayor hincapié en la privatización de servicios, prestación externa, selección de las prestaciones y capacitación a los usuarios de servi- 
cios para que adquieran estos directamente (por ejemplo, mediante el pago directo).

\section{Mayor diversidad}

- La variedad de los usuarios de servicios y su familia, en cuanto a características, condiciones y requisitos, aumenta en paralelo a la diversidad étnica, la diversidad de los hogares y la flexibilidad y el carácter "atipico" que adquieren las condiciones de empleo de las sociedades europeas.

\section{Presentación del proyecto}

Los componentes del equipo de investigación provienen de seis países: Dinamarca, Hungria, Holanda, España, Suecia y el Reino Unido. Junto con el objetivo general se plantearon una serie de objetivos específicos agrupados en cuatro epígrafes:

- Descriptivos: por ejemplo la documentación sobre la demanda, la oferta y la utilización de los servicios de atención o la descripción de la estructura, el volumen y la composición del personal.

- Analítico: por ejemplo las distintas concepciones de la atención a dependientes, según sectores y países, las causas y consecuencias de la desigual composición del personal por sexos, los modelos de atención 0 las condiciones para el desarrollo de empleo de calidad.

- Metodológicas: desarrollar métodos innovadores de investigación internacional.

- Respaldo al debate y al intercambio, mediante la creación de un directorio de iniciativas en atención a personas dependientes.

El proyecto se desarrolló en tres etapas de septiembre de 2001 a abril de 2005. La primera se centró en la descripción y revisión de los servicios de ayuda y de la atención a dependientes, dedicándose sobre todo a trabajo de despacho, de elaboración de las fuentes de datos halladas. En la segunda etapa, la más importante del estudio, se realizaron tres estudios de casos de atención a dependientes en entornos específicos: en centros con niños menores de 6 años, en ancianos y en adultos no jubilados que padecían discapacidades graves. La cuarta parte de esta etapa se ocupó del desarrollo de un método innovador de trabajo internacional en servicios prestados a niños $\mathrm{y}$ ancianos. En la tercera etapa se identificaron y recogieron iniciativas en atención a dependientes, poniendo el énfasis en la calidad, la profesionalización, el género y las conceptualizaciones.

El proyecto ha cruzado muchas fronteras. Ha sido una iniciativa internacional que ha utilizado diversos métodos, desde el análisis secundario de conjuntos de datos a estudios minuciosos de casos de cuidadores y análisis de videos sobre el concepto de atención a la dependencia. Ha sido intersectorial, cubriendo la atención a lo largo de la vida, de la infancia a la vejez. Finalmente ha sido un intento de acercar politicas, estructuras y práctica: lo habitual es que los estudios internacionales trabajen en uno o dos de estos campos, pero raramente en los tres.

Pueden encontrarse los informes sobre todas las partes de la investigación en la página web del proyecto www.ioe. ac.uk/tcru/carework.htm. 


\section{El significado de "atención a personas dependientes"}

Hasta aquí nos hemos referido a "atención", "prestación de ayuda" y "servicios de atención" como si su significado fuese evidente por sí mismo y lo entendiese todo el mundo, cosa que no es cierta. Las definiciones resultan difíciles y controvertidas: no son ni evidentes por sî mismas ni indiscutibles, entre otras razones porque sus limites no son claros ni definitivos. Muchos trabajos incluyen elementos de atención. Los limites entre atención y otros ámbitos de políticas (como por ejemplo entre atención y educación infantil o entre atención, vivienda y sanidad en adultos) son variables y tienden a aproximarse. La actividad que en un país puede tildarse de "atención a dependientes", en otro puede constituir parte de otro campo de políticas diferente como educación o pedagogía.

Los límites pueden desplazarse tanto que de hecho la atención puede desaparecer como politica y campo profesional distinto y formar parte de otro ámbito. El ejemplo más claro es la pedagogía. En el mundo angloparlante no suele entenderse en el sentido utilizado aquí, sino a menudo como la "ciencia de la educación". Sin embargo en la Europa continental tiene un significado muy diferente e influye en las politicas y en la práctica de muchos países. Adopta un enfoque global: la atención es importante pero se considera vinculada inseparablemente a la práctica educativa, evolutiva y otras. La pedagogía como teoría y práctica y el pedagogo como profesional han adquirido un mayor desarrollo y profundidad en Dinamarca en donde los "servicios de atención" se insertan en el campo de la pedagogía y se consideran de hecho "servicios pedagógicos". Desde el punto de vista danés es imposible definir un dominio de atención a parte, al menos en el trabajo con niños, jóvenes y adultos más jóvenes.
Debatir sobre el significado y las definiciones no es un mero ejercicio académico abstracto: tiene profundas implicaciones para el trabajo y la calidad del empleo. Pensemos por ejemplo en los empleados de los servicios de ayuda en centros a niños menores de 5 o 6 años en el Reino Unido y Dinamarca. Se les denomina respectivamente "cuidadores infantiles" y "pedagogos". Los pedagogos daneses son licenciados, mientras que la titulación académica de la mayoría de los "cuidadores infantiles" británicos es como máximo bachillerato. Los pedagogos daneses ganan aproximadamente el doble que los cuidadores de guardería en el Reino Unido. Los "cuidadores infantiles" británicos se limitan a trabajar con niños, mientras que los pedagogos daneses pueden trabajar con personas de cualquier edad y tienen muchas posibilidades de promoción profesional.

Tenemos ya algunas ideas sobre las condiciones que pueden facilitar empleo de "calidad". En líneas generales, los trabajos definidos como "atención" son de menor calidad que aquellos calificados como "educativos" o "pedagógicos". Las condiciones de empleo son mejores en unos países que en otros. Trataremos estos temas con mayor extensión en las conclusiones.

Ante tan distintas interpretaciones, el equipo de investigación ha considerado problemáticos los conceptos de "atención a dependientes" y "servicios de atención", cuestionándolos a lo largo del estudio y en las conclusiones. Pero teníamos que adoptar un enfoque pragmático dado que no podiamos perder el tiempo discutiendo sobre significados. Sacar adelante la investigación implicaba que debíamos ponernos de acuerdo para definir qué servicios y trabajos formaban parte del "campo de atención a dependientes", objeto de nuestro estudio. Nuestra definición se centró en los trabajadores 
remunerados de primera línea (por contraposición, por ejemplo, a los directivos), entre cuyos cometidos no entra asistir a miembros de su propia familia, que trabajaban en tres tipos de servicios de atención: infantil y extraescolar (incluidas las clases a niños que no han cumplido aún la edad de escolarización obligatoria), infantil y juvenil en internados y familias de acogida y finalmente la atención a adultos con discapacidades, incluidos los ancianos.

Dicho "dominio de la atención a dependientes" es de gran alcance, al cubrir cualquier edad y muchos tipos de entornos, desde el trabajo realizado en las casas al trabajo en toda una serie de centros de dia y en régimen residencial. Aun así quedan excluidos muchos trabajadores remunerados cuya tarea incluye aspectos de ayuda, como muchos de los empleados domésticos que forman parte de la economía sumergida. Nuestra definición excluye también a muchas personas que prestan ayuda "informal" de forma altruista que, como demuestra claramente la investigación, se ocupan de la mayor parte de la atención a dependientes en Europa.

\section{Tipologia del personal}

Al abordar los tipos de trabajo en el dominio de la atención a dependientes de los seis países colaboradores en la investigación "La atención a personas dependientes en Europa” se desarrolló una tipología de tres niveles según el grado de estudios inicial. En el nivel inferior (el grado más bajo de estudios, es decir enseñanza primaria) hay un grupo de trabajadores, muchos de los cuales desempeñan su actividad en el medio doméstico (por ejemplo cuidadores familiares, cuidadores de dia familiares y asistentes personales con sistema de "pago directo"). En el nivel superior (el grado superior de educación), están los pedagogos y profesores infantiles. En el nivel intermedio (bachiller) están algunos de los cuidadores infantiles y los que trabajan con grupos de adultos.

Aquellos que trabajan con niños y jóvenes tienen por lo general mayor nivel de estudios que quienes trabajan con ancianos (la diferencia sigue aumentando). Los profesionales de la atención a dependientes tienen nivel educativo superior en Dinamarca, en donde los pedagogos (muy preparados) trabajan con todos los grupos de edad y el resto de trabajadores tiene un nivel de formación relativamente elevado. La mayoria del trabajo de atención a dependientes lo prestan mujeres que (como en la población trabajadora general) tienen entre 25 y 44 años (por lo que probablemente tengan además sus propias responsabilidades de atención). No fue posible hallar información similar sobre otros aspectos importantes tales como los étnicos o las responsabilidades de los trabajadores en materia de atención.

El nivel de trabajo a tiempo parcial supone más de la mitad de las personas que trabajan en la atención a dependientes, reflejo de la elevada proporción femenina. Como en el resto de la población trabajadora general, el trabajo a tiempo parcial en el campo de la atención a dependientes está extendido en Holanda y el Reino Unido y es raro en Hungría y en España. Los sueldos varian en función del grupo de trabajadores y de los países, si bien hay una clara relación entre el nivel de educación inicial y el nivel salarial: en Dinamarca es alta en ambos y en el Reino Unido baja.

Resultó imposible obtener un panorama fiable y global del volumen del personal que dedicado a la atención a dependientes, objeto del proyecto en los seis países participantes, por no hablar del 
conjunto de los Estados miembros de le UE. La mejor fuente de comparación, la Encuesta Europea de Población Activa, resultó inadecuada a la postre. De entre los países participantes Dinamarca dispone de la información más detallada sobre el personal que se dedica a la atención a dependientes, información que indica que este país cuenta con más personal dedicado a la atención (relativo a la población general): aproximadamente el $10 \%$ de la población activa total está empleada en el sector de la atención a dependientes. Dicha proporción puede ser parecida o ligeramente inferior en Suecia, pero en los demás países participantes, que en general disponen de un nivel inferior de servicios, es sustancialmente menor: en torno al 7\% en Holanda, $5 \%$ en el Reino Unido y 2-3\% en España y Hungria (las cifras son aproximadas, no medidas exactas ni comparables). La mayoría de los trabajadores daneses lo son a tiempo completo, mientras que en Holanda y en el Reino Unido gran parte ejerce a tiempo parcial.

No hay dudas sobre la de escasez real o previsible de personal en el conjunto de países participantes. La situación parece especialmente preocupante en Hungría y el Reino Unido, en los que la remuneración es muy baja. Dinamarca ha abordado la escasez de profesionales de la atención a dependientes debidamente preparados, los pedagogos, aunque tiene escasez en el grupo de personal menos formado: los auxiliares de asistencia social y sanitaria. Al mejorar el nivel educativo de la población, cada vez resultará más dificil reclutar estudiantes para este trabajo menos cualificado.

Se han propuesto o aplicado distintas medidas para hacer frente a la falta de personal, entre ellas la mejora del nivel de formación y profesionalidad (por ejemplo en Dinamarca y Suecia), cambiar las estrategias de reclutamiento, sobre todo de grupos escasamente representados (como en Holanda y en el Reino Unido), mejorar las condiciones de empleo, prolongar la vida laboral del personal y otras medidas tales como campañas mediáticas que mejoren la imagen pública de la atención a dependientes.

\section{El sesgo del personal en cuanto a género}

Por lo general el personal dedicado a la atención a dependientes es casi todo femenino. Hay alguna variación en función de los grupos de edad de las personas objeto de atención: hay más varones trabajadores cuando se trata de la atención a jóvenes y adultos más jóvenes. Aunque las personas entrevistadas en la investigación (profesionales, profesores y directivos) creían que era una buena idea que hubiera más varones en la atención a dependientes, no es algo prioritario en las políticas o en la práctica. De hecho en España las politicas dirigidas al desarrollo de la atención a dependientes constituyen una estrategia para aumentar el trabajo femenino, que está por debajo de la media europea.

Una consecuencia de la descompensación de género en esta actividad es la baja remuneración, si bien el sueldo no es una de las causas de la descompensación de género: a pesar de que en Dinamarca y en Suecia la remuneración sea más elevada que en los demás países, pocos varones se dedican al cuidado y atención infantil. Como mucho es probable que la baja remuneración forme parte de un proceso más amplio por el que la formación y el empleo reproducen un sesgo de género en el personal. Dicho de otro modo, se da por supuesto que hay unas estructuras de enseñanza, empleo y práctica típicamente femeninas, lo que a su vez atrae a las mujeres y disuade a los varones. 
Esta descompensación de género en el personal también parece vincularse con el predominio de una concepción de la atención como eminentemente femenina, réplica del carácter también femenino del cuidado doméstico. Según esta lógica la mujer tiene una capacidad innata para el cuidado o la desarrolla a través del trabajo doméstico, por lo que está equipada intrínsecamente también para la atención remunerada y sin necesidad de una gran formación. Es este modo de pensar el que inspira el desarrollo posbélico de servicios domiciliarios para personas mayores en Suecia, en donde las amas de casa experimentadas se consideraban al principio las más adecuadas como cuidadoras remuneradas. Aunque, como ocurre en todas partes, esta concepción de la atención a dependientes (como sustituto del cuidado doméstico) haya cambiado, en realidad sigue profundamente enraizada en las sociedades, razón por la cual el cambio de percepción tardará mucho tiempo en impregnar una actividad menos sesgada con respecto al género.

\section{El concepto de atención a dependientes}

Ya hemos abordado cómo hay que interpretar que una misma área de trabajo afecte a campos tan distintos como el de las políticas, los recursos o la práctica. Es más evidente en servicios para niños que pueden considerarse como "cuidado infantil", "educación" o "pedagogia”. En algunos países participantes como Dinamarca, España o Suecia (en los que estos servicios tienen un cariz principalmente educativo o pedagógico) "el cuidado infantil" (como ayuda a los padres trabajadores) se reconoce como función de los servicios pero no la principal o única función. No obstante, aun en aquellos países en los que todos o parte de los servicios siguen inspirándose en el concepto de "cuidado infantil", dicho concepto incorpora progresivamente un rol evolutivo o educativo, si bien las estructuras (sobre todo los empleados) siguen ancladas en el miope concepto original de cuidado parental sustitutivo.

En algunos países hallamos otros servicios en campos de atención más específicos y explícitos, como ocurre en Suecia y en el Reino Unido, en los que la prestación de servicios a adultos se denomina "atención social". Pero en el estudio de casos los sujetos tienen dificultades para definir el concepto de atención 0 atención social. Esta última, por ejemplo, se reduce con frecuencia a una etiqueta descriptiva referida a un conjunto de servicios, mientras que la atención puede definirse a veces en función de lo que no es (por ejemplo, que no es sanitaria ni trabajo social). El concepto de atención puede no obstante deducirse observando en los servicios analizados las conversaciones de especialistas y otras personas a cerca del trabajo. Aparecen distintos conceptos de atención: dar protección, fomentar la autonomía y la inclusión, crear un entorno similar al familiar, atención como artículo de primera necesidad o servicio que se puede comprar y vender. Estos conceptos no tienen por qué excluirse necesariamente: la idea de atención puede integrar una variedad de conceptos y además cambiar con el tiempo.

Hay distintos conceptos para definir quién es el trabajador. En los servicios infantiles, como ya hemos visto, el trabajador puede ser un "cuidador infantil", "pedagogo" o "profesor". En los servicios de adultos, lo trabajadores son pedagogos, enfermeros (se incluye a los auxiliares de enfermería), terapeutas u otro tipo de profesional. Muchos de los que ejercen la atención a dependientes tienen distintas etiquetas. Un avance potencialmente importante, dado el crecimiento de los 
planes salariales directos, es un nuevo tipo de trabajador, el "asistente personal", muy próximo a la idea de atención como artículo de primera necesidad o servicio, utilizada por la persona que precisa atención (es decir el empleador) para dotar a la gestión de servicios específicos.

¿Hasta que punto estas diferencias conceptuales y estructurales influyen en la práctica real? Se trata de un área amplia e importante aunque poco explorada. Una parte del proyecto tenía que desarrollar un método audiovisual para analizar el concepto de ejercicio profesional: Sophos (Programa de observación fenomenológica de segundo orden ${ }^{1}$ ). Aunque el trabajo era básicamente metodológico, proporcionó ejemplos del tipo de hallazgos que podia aportar el método, apuntando diferencias de concepto importantes.

Cuando los observadores daneses, desde su óptica pedagógica, examinaron sendos videos de centros infantiles (danés, inglés y húngaro respectivamente), observaron tres "lógicas" o razonamientos (escuela, hogar/familia e infancia) y tres espacios de vida cotidiana (aprendizaje organizado, vida diaria y juego). Comprendieron que el centro danés tenía una lógica de infancia, cuya meta es que los niños adquieran experiencia y lleguen a descubrimientos por sí mismos. También consideraron que los tres espacios eran igualmente importantes, percibiendo que en conjunto se trataba de trabajo pedagógico (más que de cuidado).

Sin embargo, al examinar los videos de los otros dos países apreciaron una lógica y un tipo de espacio distintos. En la pelicula inglesa observaron una prác- tica imbuida de lógica escolar. El día a día gravitaba en un espacio de aprendizaje organizado, muy controlado por los adultos y muy estructurado. Por el contrario, los observadores daneses vieron que el film húngaro presentaba un centro cuya práctica estaba gobernada por la lógica del hogar/familia, en el que el cuidador actuaba como madre y educador. Los hechos se centraban en el espacio de la vida cotidiana, en el que el ideal es la buena familia y el niño bien educado. En su opinión la práctica danesa también daba prioridad a las actividades cotidianas pero desarrolladas de un modo distinto a la vida familiar. También notaron que en la guardería húngara dominaba la mujer.

Desde el punto de vista del investigador danés, los observadores ingleses (cuidadores y expertos en la infancia) parece que aprecian como valor fundamental la competencia e independencia (capacidad de decisión) del niño. La utilización de conceptos como elección y libertad del niño o el niño como ser humano independiente, desde la perspectiva danesa denota un punto de vista liberal sobre el fenómeno. Los observadores daneses aluden a la autonomía y autogobierno del niño, pero dichos conceptos están más próximos a la interdependencia que a la independencia, reflejo tal vez del concepto europeo continental del ser humano competente que sólo es libre si mantiene una relación equilibrada con la comunidad (es decir, interdependencia).

A pesar de las diferencias, pueden distinguirse muchos aspectos comunes, compartidos por países y sectores que, como explicaremos posteriormente, acarrean consecuencias importantes para

\footnotetext{
${ }^{1}$ Nota del traductor: el acrónimo Sophos procede de las iniciales de los términos en inglés: Second Order Phenomenological Obseration Scheme.
} 
las politicas futuras. En general, en todos los sectores el trabajo se vuelve cada vez más complejo y exigente como consecuencia del contexto cambiante señalado anteriormente y de la mayor toma de conciencia de lo que supone responder de un modo global a las necesidades de los usuarios de servicios de atención. Pueden identificarse algunos requisitos del trabajo comunes a todos los sectores y países:

- Satisfacer las necesidades fisiológicas fundamentales y de protección de los destinatarios.

- Fomentar el desarrollo y la autonomía.

- Favorecer la relación integradora entre la persona, la familia, los amigos y comunidades más amplias (trabajar con autonomía $y$ solidaridad, autonomía $e$ interdependencia y fomentar la inclusión y la ciudadanía).

- Aspectos relacionales: comunicación, escucha, empatía, estar en relación con los demás.

- Establecer una red de contactos (con la familia y la comunidad) y trabajo en equipo (con otros trabajadores y servicios).

- Reaccionar al cambio de imagen de la persona objeto de atención (por ejemplo, de objeto pasivo a sujeto activo con derechos).

- Capacidad para reciclar los conocimientos, para implicarse en el proceso continuo de adquisición de conocimientos, identidad y valores.

- Desenvolverse en la diversidad.

A su vez son necesarias competencias $y$ cualidades comunes en los cuidadores, con independencia del sector:

- Competencia comunicativa (en muchos idiomas, con muchas personas y organizaciones, sin olvidar la escucha).

- Competencias analíticas y de reflexión.

- Comprensión y valoración del aprendizaje como proceso continuo.

- Competencias y experiencia personales (como paciencia, voluntad de profundizar en las cosas, empatía, desafio) y capacidad de conectar lo personal y lo profesional.

- Conocimientos profesionales (sobre el grupo específico con el que opera el cuidador y sus necesidades particulares, sobre ciencia social/psicología social: un ejemplo sería el desarrollo de una red de contactos sociales).

- Juicio contextualizado.

- Saber manejar teoría y práctica.

- Competencias musicales y estéticas.

- Amplia cultura general.

- Competencias en prevención de tensiones psicosociales y físicas, en especial cuando se trabaja solo (por ejemplo conocimientos de ergonomía o de primeros auxilios).

- Competencias interculturales (y de otro tipo de diversidad).

- Competencias en trabajo interprofesional y trabajo en equipo.

Los requisitos y las competencias 
ponen de manifiesto que "la atención a dependientes" no puede sustituir al trabajo realizado en el hogar por los miembros (principalmente femeninos) de la familia, pues entraña relaciones, prácticas y posibilidades diferentes y exige capacidades diferentes o especificas. Además, complementa más que sustituye, pues el cuidador no sustituye a la madre 0 a otro miembro femenino de la familia, ni el hecho de ser mujer o ama de casa garantiza que se tengan las competencias necesarias. Sin embargo los cuidadores sí aportan al trabajo la experiencia personal (de todo tipo), que constituye un recurso y una competencia, aunque no sea ni homogéneo ni indispensable.

\section{¿Es la atención un empleo de calidad?}

Merced a la abundancia de publicaciones, entre ellas las directrices europeas, podemos glosar una serie de características, de amplia aceptación, que definen el empleo de calidad. Entre ellas están el sueldo, las prestaciones, la formación inicial y continuada, un entorno favorable, la salud y la seguridad, el futuro profesional, la autonomia, la toma de decisiones, un empleo coherente, el reconocimiento y el estatus social, la igualdad de oportunidades y no discriminación y la conciliación familia-trabajo.

Las conclusiones del proyecto demuestran en general que por una parte (salvo ciertas excepciones) el sueldo y otras condiciones laborales son malos, la formación inicial y posterior son variables, las oportunidades de desarrollo profesional están limitadas y el trabajo es con frecuencia exigente en el sentido físico (como levantar pesos o soportar ruidos) y psicológico. Por otra parte los sujetos manifiestan que están satisfechos con el trabajo (concretamente, se sienten a gusto con sus compañe- ros), que suelen tener bastante control sobre el trabajo y las decisiones (aunque no sobre los presupuestos) y que no les resulta difícil conciliar trabajo y vida familiar. Con respecto a esto último, la atención remunerada a tiempo parcial 0 en horas atipicas ha permitido a la mujer administrar la vida laboral y familiar en ausencia de una infraestructura sólida de apoyo público a las personas que se dedican a la atención. Cuando existe tal apoyo, como en Dinamarca o Suecia, las responsabilidades en el trabajo son más fácilmente compatibles con una jornada laboral más larga y un empleo continuo. Es decir, que si el empleo en sí puede ayudar u obstaculizar a las personas que se dedican a la atención, otro tanto pueden conseguir las politicas públicas (o su ausencia).

La mayoría de los trabajadores creen que la atención tiene un bajo estatus, a pesar de no albergar dudas sobre la importancia de su trabajo, su valor y coherencia. La principal excepción a esta descripción la constituyen los pedagogos daneses que, en general, consideran que el estatus social del trabajo pedagógico es bastante elevado actualmente (ha mejorado en los últimos años, en los que esta carrera atrae a los jóvenes). Otro asunto importante más a largo plazo es la postura del creciente número de "asistentes personales" y hasta qué punto el crecimiento en esta área puede influir en el estatus social de la atención a adultos con graves discapacidades y a ancianos. Mientras este desarrollo aumente las posibilidades de elección y control de aquellas personas que puedan adquirir los servicios que deseen, los "asistentes personales" pueden acabar siendo vistos como una nueva clase de empleados, con escasas posibilidades de formación y desarrollo y fáciles de explotar. Aunque no sea un resultado inevitable, habrá que estar muy pendiente para evitar que suceda. 
En consecuencia, el equilibrio entre exigencias, libertad de decisión y recompensa es tal que la mayoría de los trabajadores son optimistas sobre su trabajo aunque admitan la existencia de características negativas y que, con notables excepciones, el trabajo está aún lejos de ser un empleo de calidad.

\section{Perspectivas futuras}

Para concluir abordaremos algunas de las posibles perspectivas futuras de la atención a dependientes, como la estructura del cuidador futuro, la posibilidad de que "la atención a dependientes" siga siendo una política y un campo de empleo independiente y las condiciones que hacen falta para crear empleo de calidad y conseguir diversidades en el personal. Terminaremos preguntando si no habrá crisis de la atención y, si es así, cuál debería ser la respuesta de las sociedades europeas.

\section{¿Cómo podría estructurarse el personal?}

Ante la complejidad de las exigencias y la evolución del contexto en el que se desarrolla la atención a personas dependientes, sea del tipo que sea, concluimos que es necesario reducir de tres a dos los niveles de la estructura actual de personal, eliminando paulatinamente el nivel inferior (sin finalizar la educación secundaria). Los dos niveles estarian constituidos respectivamente por profesionales licenciados universitarios preparados y por otros trabajadores con educación secundaria o bachiller, que trabajarian juntos. El nivel educativo de acceso debe completarse con formación continua y desarrollo profesional (es decir, comprometerse a un aprendizaje continuado en instituciones de formación). Ha de haber diversidad en la composición del personal, tanto en lo que respecta a género y edad, como desde el punto de vista étnico, y disponer de oportunidades de movilidad horizontal y vertical (esto es, poder cambiar entre sectores estrictamente definidos y tener la posibilidad de ascender profesionalmente).

Esta afirmación manida nos enfrenta a una serie de preguntas importantes. ¿Cuál debe ser el equilibrio entre los dos niveles de titulación, profesionales y otros trabajadores? ¿Cuál debe ser la base profesional, generalista o especialista? Es decir, ¿basta con una formación básica que capacite a los trabajadores para operar en una amplia gama de grupos y contextos o debe exigirse una formación más especializada que capacite a los trabajadores para actuar con grupos concretos en un número limitado de contextos? Si se opta por lo primero, ¿cuál debe ser la disciplina de referencia?

La respuesta a estas preguntas probablemente esté condicionada por la tradición existente en cada país, el concepto que se tenga del trabajo y la filosofía general sobre política de bienestar. Por ejemplo la respuesta de la tradición pedagógica, con su perspectiva global, en combinación con un régimen de seguridad social universal debidamente financiado, como ocurre en Dinamarca, es dotarse de un gran número de profesionales que se encarguen de todas las actividades de atención a dependientes, con un enfoque generalista fundamentado en la disciplina de la pedagogía y aplicado a una gran variedad de personas, desde niños pequeños a ancianos que padecen demencia. Por poner otro ejemplo, uno de los participantes holandeses en la investigación, al reflexionar sobre la situación en Holanda, era también partidario de una profesión generalista, aunque se imaginaba a este grupo profesional más reducido, más burocrático y de una naturaleza híbrida, combinación de pedagogia, trabajo social y sanidad. 
¿Es la atención a dependientes un campo de trabajo en si mismo?

Hemos sostenido desde el principio que el término y el concepto de "atención a dependientes" no deben darse por supuestos y que está demostrado que los limites entre "atención" y otros campos son cada vez más imprecisos, lo que plantea la siguiente pregunta: ¿debe ser la "atención a dependientes" un área distinta de política, financiación y práctica, en la totalidad o en parte, del campo que hemos definido como "dominio de la atención a dependientes"? Efectivamente, podemos distinguir al menos tres enfoques diferentes en el campo de la atención a dependientes y su personal, según sea la respuesta a dicha pregunta:

- Enfoque no específico de "atención". Esta se integra en un campo más amplio en el que el personal se desenvuelve con muchos grupos de distinta edad (aunque pueda haber cierto grado de especialización durante la formación inicial o posterior). El paradigma es Dinamarca, país en el que ser pedagogo es una profesión muy importante y de amplio espectro que permite trabajar con personas de cualquier edad (en menor medida con ancianos) en muchos contextos. Casi todos los servicios del "campo de atención a dependientes" se integran en un campo pedagógico más general, que tampoco coincide con el campo educativo.

- Enfoque de "atención" independiente. En el extremo opuesto del espectro, la "atención" se constituye en un campo en sí mismo, aunque muy relacionado con otros, cuyo personal se estructura laboralmente en torno a diferentes grupos. Es el caso de Holanda, en la que las tres par- tes de nuestro campo de atención están cada vez más diferenciadas. Los servicios para adultos, ancianos incluidos, se denominan "atención a largo plazo" y están próximos a la asistencia sanitaria. La atención en régimen interno a niños y jóvenes, junto con actividades con otros jóvenes, se consideran "prestaciones de seguridad social", con un fuerte componente pedagógico, mientras que los servicios de "cuidado y atención infantil" tienen otra orientación en cuanto a politica y legislación, con un enfoque evolutivo y de mercado laboral. A pesar de compartir el mismo marco de formación, el campo de la atención a dependientes aglutina grupos diferentes de trabajadores que se forman a diferentes niveles y para diferentes especialidades.

- Enfoque mixto, en el que determinadas parcelas de actividad en el "campo de la atención a dependientes" se integran en un campo más amplio, mientras otras permanecen en otro campo de atención y el personal se estructura en torno al trabajo con diferentes grupos de edad. Suecia es un ejemplo de este sistema, dado que tiene diferentes orientaciones para la atención infantil y la atención adulta (pedagogía y atención social respectivamente) y diferente dependencia ministerial (Educación y Seguridad Social). Por eso el personal que trabaja con niños en edad preescolar, el que trabaja en los servicios de atención extraescolares y los maestros han sido agrupados en un marco conjunto de formación y serán considerados en el futuro profesores, quedando todos los servicios acogidos al sistema de educación: la atención se integra pues en el campo de la educación (que a su vez adopta un enfo- 
que amplio que debería denominarse pedagógico). Tal reestructuración crea una profesión única para el trabajo en los servicios de día con niños y jóvenes (no con adultos ni en los servicios de atención a niños y jóvenes en régimen interno).

Hay que destacar que eliminar la "atención" como campo independiente en materia de política, presupuesto y práctica no significa dejarla fuera de la politica, los presupuestos y la práctica. La "atención" por ejemplo constituye un elemento importante del campo pedagógico, mientras que el aumento de la investigación sobre la "ética de la atención" nos recuerda que la "atención" puede conceptualizarse como una ética susceptible de afectar a un amplio abanico de ocupaciones y no únicamente en lo que hemos definido como "campo de atención a dependientes". En resumen, la "atención" en los servicios no depende de la existencia de los "servicios de atención" (por ejemplo el personal de los colegios, hospitales, prisiones o cualesquiera otras instituciones pueden trabajar en "atención" entendida como "ética de la atención") en mayor medida que el trabajo en atención precisa de personal específico.

\section{¿Cuál es el potencial de la atención a dependientes como fuente de empleo de calidad?}

La diferencia en volumen y calidad global del trabajo en el "campo de la atención a personas dependientes" (objeto del proyecto) entre países como Dinamarca y Suecia por una parte y España y Hungria por otra, indica en qué medida la atención a dependientes contribuye a la creación de empleo de calidad en la Unión Europea. Considerando los países y sectores que tienen empleo de calidad, ¿qué condiciones reúne dicho empleo? Entre ellas hay que señalar:
- Unos servicios debidamente financiados. Hasta la fecha tales servicios se han financiado en los países nórdicos mediante una elevada imposición, obviando otras alternativas.

- La valoración social de la población objeto de atención. La situación de las personas que trabajan actualmente con niños mejora debido en parte a la valoración social y política de estos últimos. No ocurre otro tanto con la atención a personas mayores por la escasa valoración que merecen y el prejuicio ampliamente extendido de la "falta de resultados".

- La organización del personal con el fin de dotarlo de una expresión pública sólida y bien articulada, así como la capacidad de presión a través de los sindicatos y asociaciones profesionales para mejorar las condiciones laborales. Importa en este caso que los sindicatos y otras organizaciones profesionales no se queden sólo en el salario y en las condiciones laborales, sino que incluyan también la educación, la concepción social del trabajo, el desarrollo de politicas, etc.

- Potenciar la visibilidad del trabajo. Es necesario que haya un reconocimiento público de su complejidad y del elevado nivel de competencia que exige su desempeño adecuado.

- Desarrollo de "instituciones educativas" que asuman y fomenten el aprendizaje continuado y estimulen la reflexión y el debate libres y criticos.

- El empleo del sector público o la financiación pública deben exigir el marchamo de empleo de calidad. Las administraciones públicas jue- 
gan un papel fundamental como instancias financiadoras, reguladoras y en algunos casos proveedoras.

- La (re)conceptualización del empleo en el "campo de la atención a dependientes" como algo diferente a atención. Cuando la "atención a dependientes" es considerado como un campo distinto, la formación, la remuneración y otras condiciones suelen ser malas, pero si se la considera y define como parte de un campo más amplio o diferente (como la pedagogía o la educación) mejora notablemente la calidad del empleo. Decir esto supone reconocer que la relación no es simple ni directa sino que implica, por ejemplo, procesos complejos de reelaboración y reestructuración a largo plazo.

Hay que añadir una última condición: es probable que el empleo de calidad se desarrolle si hay un amplio reconocimiento público de que la calidad del empleo puede evitar la escasez de personal. Un debate que subyace a la orientación futura de la atención es la posibilidad de una "crisis de atención" en Europa.

\section{¿Se enfrenta Europa a una crisis en la atención a dependientes?}

Como otros estudios internacionales, este demuestra la escasez real o previsible de personal que preste servicio en el "campo de atención a dependientes". Pueden considerarse problemas transitorios del mercado de trabajo consecuencia de las condiciones locales particulares 0 reflejo de un fenómeno más profundo y duradero (una crisis emergente de atención). Cuando se produce una crisis, afecta tanto a la atención informal como a los servicios formales, cuyas causas lo son por partida doble: aumento de la demanda de atención cuando disminuye la oferta tradicional de atención (es decir, la mujer con bajo nivel educativo).

- Si tomamos el grupo de edad entre 25 y 64 años y dado el relevo de las generaciones de menor nivel educativo por generaciones jóvenes de mayor nivel... podemos dar por hecho que la escasez de personal de bajo nivel educativo constituirá un gran problema... (Esta escasez) comprometerá las inversiones y acarreará graves dificultades de dotación de personal en aquellas organizaciones laborales que funcionan con estrategias de baja capacitación y bajos sueldos...

- La lección que habría que extraer es que siempre que el listón en cualquier categoría laboral sea "mujer poco cualificada en torno a los 30 años", habrá que aumentar inexcusablemente la calidad del puesto de trabajo para que puedan aceptarlo personas de mayor nivel educativo. Si no se consigue mejorar la profesionalización del puesto, se producirá una grave escasez en la dotación de personal.

Dicho de otro modo, el breve periodo histórico durante el cual prevaleció el modelo del varón como sostén de la familia, se ha visto superado por la realidad.

¿Quién se ocupará pues de la atención en la Europa del futuro, ya sea en el hogar o fuera del mismo? Pueden concebirse diversas salidas. Algunas medidas (como los permisos parentales y los incentivos a familiares por ejercer como cuidadores) pretenden estimular la débil oferta en atención informal, sobre todo en la familia y de una generación a otra, en respuesta al "déficit de ayuda".

Otra medida consiste en aprovechar más las bolsas de cuidadores, infrautilizados actualmente, como por ejemplo volver a utilizar mujeres con bajo nivel edu- 
cativo dispuestas a trabajar por un sueldo bajo, procedentes de la inmigración actual o futura, empleadas en trabajos normalizados o en trabajos marginales de economía sumergida como la asistencia doméstica. Otra posibilidad es fomentar una mayor participación de los varones empleados en atención formal e informal. Si no mejora la cifra de los que trabajan en atención remunerada, el cambio de la atención informal a la formal sólo conseguirá feminizar la atención, dado que los varones participan más en la atención informal que en la formal. Dicho de otro modo, hay que abordar el reclutamiento de personal para ambos tipos de atención para no reproducir o agravar el desequilibrio actual (personal básicamente femenino en esta ocupación).

La tercera respuesta implica un esfuerzo sostenido para reestructurar y revalorizar la atención a dependientes con profesiones nuevas y mejorar la formación partiendo de un mayor nivel de educación inicial y siguiendo con la formación continua "en el trabajo" (brindando a los trabajadores la posibilidad de mejorar su formación).

Dichas opciones plantean cuestiones de viabilidad y de dimensión temporal. Por ejemplo, hay un gran potencial de reclutamiento de trabajadores varones, pero para conseguirlo hace falta por parte de todas las partes implicadas (instancias públicas, educadores, empresas suministradoras, sindicatos, etc.) un compromiso continuado, un análisis minucioso y una gran atención a la práctica. Se tardará en conseguir (superar una situación en la que sólo el 15 o 20\% del total de personal son hombres puede llevar un decenio).

Decidirse por las opciones señaladas plantea también una cuestión de principio y un objetivo general. ¿Es compatible con el fomento de la igualdad de género potenciar la atención informal? Aunque se puedan contratar trabajadores, ¿podría mantenerse el bajo estatus de la atención? También surgen cuestiones de orden práctico. Si es posible estimular la atención informal ¿en qué condiciones? ¿Es compatible con los objetivos de aumentar el empleo femenino? Tal vez haya que aumentar la remuneración (las prestaciones que reciben los padres con licencia parental) y aun así sea menos probable que las mujeres (o los varones) con mayor nivel de estudios y mejores puestos de trabajo respondan a tales medidas (es probable que sean las mujeres de bajo nivel educativo las que se tomen periodos más largos de permiso parental). ¿Es posible reclutar más trabajadores entre los grupos menos representados? En ese caso, ¿cómo? ¿Serán las sociedades las que paguen los costes de una atención más profesionalizada?

En la práctica las opciones que se han planteado no se excluyen mutuamente. Cada una tiene algo que aportar. La reestructuración y revaloración del trabajo en el campo de la atención a dependientes atraerá candidatos con mejores niveles educativos. Hay razones y necesidad suficiente para la incorporación de trabajadores varones en todos los niveles, así como es probable que siga haciendo falta, aunque menos, personal con menor nivel de estudios, que requerirá un mayor reclutamiento entre los grupos menos representados (y mayor atención al desarrollo profesional de estos trabajadores). A medida que se retrasa la edad de jubilación en toda Europa, habrá personas de más edad que se sentirán atraidas por la atención a dependientes, al aumentar las oportunidades de formación y una mayor flexibilidad del horario laboral. No hay motivos de preocupación pues ante la crisis de prestación de ayuda que está surgiendo en Europa: es también una oportunidad para dotar a la atención a dependientes de la consideración y el valor que se merece. 